\title{
Biopolymer-Based Coatings and Packaging Structures for Improved Food Quality
}

\author{
Amparo Lopez-Rubio, ${ }^{1}$ Maria J. Fabra, ${ }^{1}$ Marta Martinez-Sanz, ${ }^{1}$ \\ Sandra Mendoza, ${ }^{2}$ and Quan V. Vuong ${ }^{3}$ \\ ${ }^{1}$ Food Safety and Preservation Department, IATA-CSIC, Avda. Agustin Escardino 7, Paterna, 46980 Valencia, Spain \\ ${ }^{2}$ Departamento de Investigación y Posgrado en Alimentos, Facultad de Química, Universidad Autónoma de Querétaro, \\ 76010 Querétaro, QRO, Mexico \\ ${ }^{3}$ Nutrition Food \& Health Research Group, School of Environmental and Life Sciences, University of Newcastle, P.O. Box 127, \\ Ourimbah, NSW 2258, Australia
}

Correspondence should be addressed to Amparo Lopez-Rubio; amparo.lopez@iata.csic.es

Received 20 November 2017; Accepted 28 November 2017; Published 14 December 2017

Copyright (C) 2017 Amparo Lopez-Rubio et al. This is an open access article distributed under the Creative Commons Attribution License, which permits unrestricted use, distribution, and reproduction in any medium, provided the original work is properly cited.

Biopolymers, that is, polymers derived from bio-based resources, have a central role in keeping or improving food quality. They have mainly been used as structuring or texturizing agents in food, but their potential as edible coatings for increasing postharvest shelf-life of fresh produce and as bio-based packaging structures for substituting petroleumbased polymers is gaining importance. Therefore, the aim of the present special issue was to compile recent developments in these two areas. A substantial number of papers were submitted, highlighting the research interest in these topics and, after a thorough peer review process, four papers were selected to be included in this special issue. Three of them show recent advances in the development of edible coatings for improving postharvest quality of fresh fruits and vegetables, while the other research work deals with the development of a bio-based structure for food packaging applications.

The manuscript of A. Ullah and coworkers reports about the evaluation of three different edible coatings for enhancing quality during storage of the vegetable bell pepper. Their results show that Gum Arabic has an excellent potential as an edible coating for the studied cultivar, as it significantly reduced weight loss, membrane leakage, chilling injury, and decay incidence, without affecting the visual appearance in terms of colour of the fruit.
Another gum with potential as an edible coating is guar gum, as shown in the paper of X. Ruelas-Chacon and coworkers. They evaluated plasticized guar gum coatings on the postharvest and sensorial quality of Roma tomatoes stored at $22^{\circ} \mathrm{C}$. Interestingly, it was found that, apart from improving firmness, reducing weight loss, and delaying several decay parameters, the application of guar gum positively affected the sensorial properties of the tomatoes as demonstrated by the results from a trained panel.

The research work of S. Gunaydin and collaborators deals with the development of active composite edible coatings for improving postharvest quality of plums during cold storage. The strategy in this case was to combine hydroxypropyl methylcellulose (HPMC) as the structuring agent of the coating with a beeswax that provided hydrophobicity, thus reducing weight loss and incorporating three different antifungal agents with proven activity. The stability and applicability of the emulsion coatings were improved through the addition of stearic acid and glycerol, respectively. Important quality attributes of the fruits were not adversely affected by the coatings, while their application led to better control of weight and firmness loss and they contributed to a reduction in physiological disorders such as flesh browning and bleeding.

Finally, the research work from Z. Wang and collaborators deals with the development of bio-based hybrid films for food 
packaging applications. The authors studied how addition of sodium alginate modified the physical-chemical properties of cast collagen films. They report that incorporation of the carbohydrate polymer improves the thermal stability of the collagen films which also showed increased tensile strength and improved barrier to water vapour, although no differences were observed in the oxygen permeability of the films. Moreover, the components seemed to be compatible as no phase separation was observed in the blends.

We hope that the readers enjoy the reading and that this compilation of papers serves as an inspiring basis for investigation in this exciting and promising research area.

\section{Acknowledgments}

We would like to sincerely thank all the authors who contributed to this special issue, as well as the expert reviewers who provided constructive feedback and extremely useful comments.

Amparo Lopez-Rubio

Maria J. Fabra

Marta Martinez-Sanz

Sandra Mendoza

Quan V. Vuong 

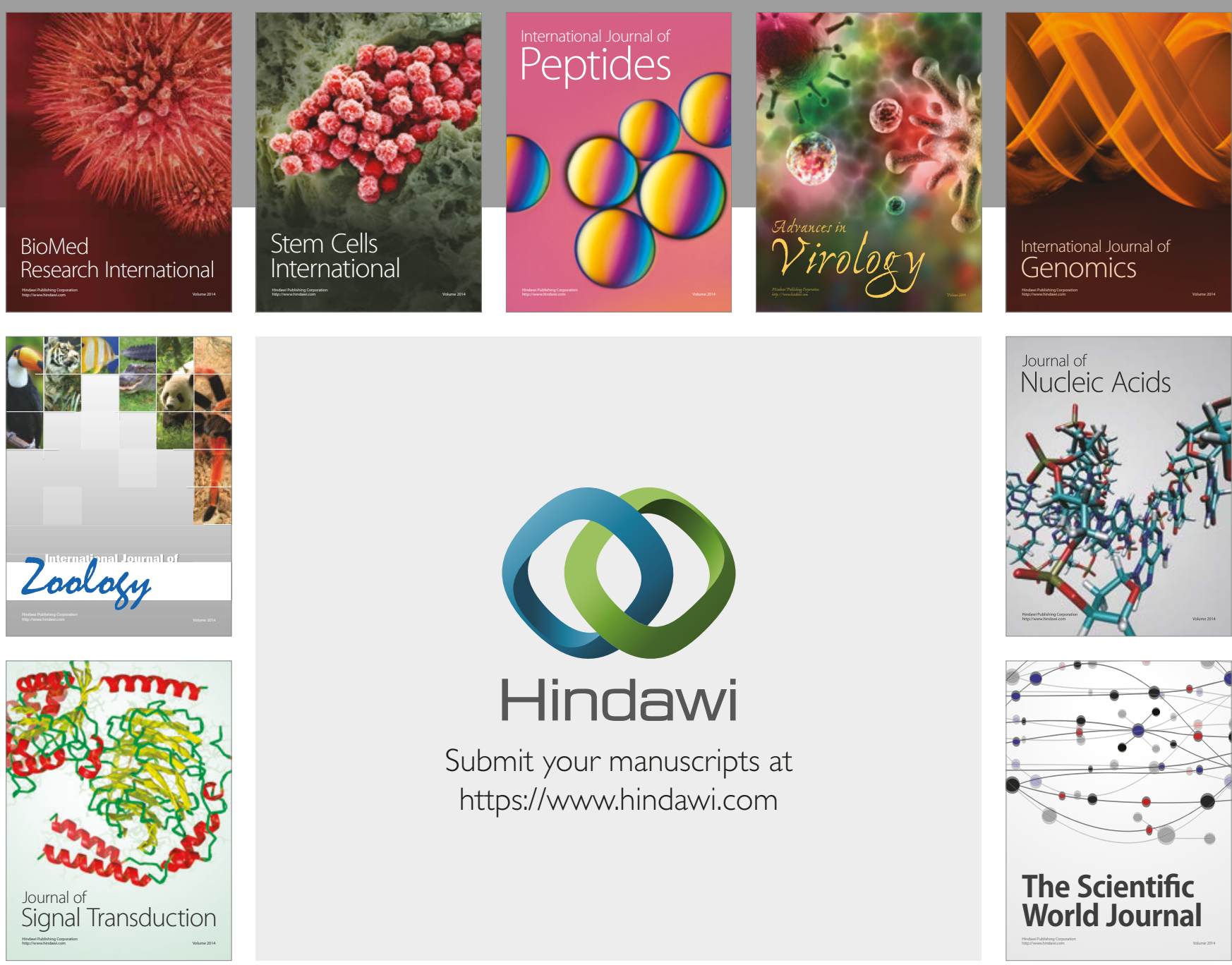

Submit your manuscripts at

https://www.hindawi.com
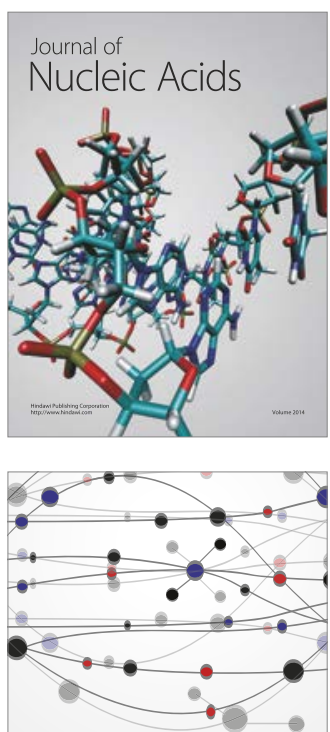

The Scientific World Journal

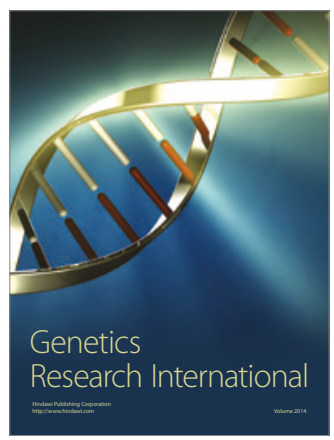

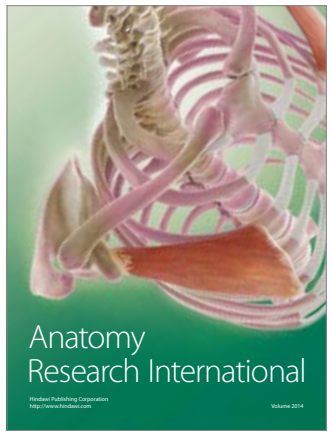

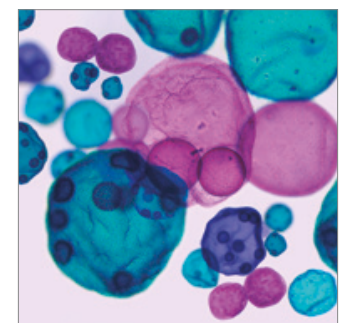

International Journal of Microbiology
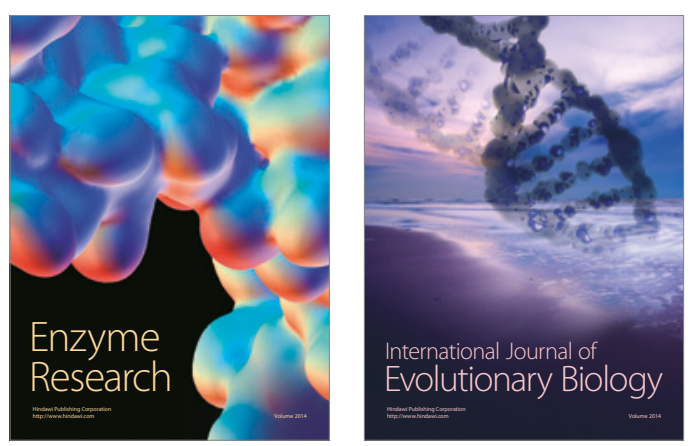
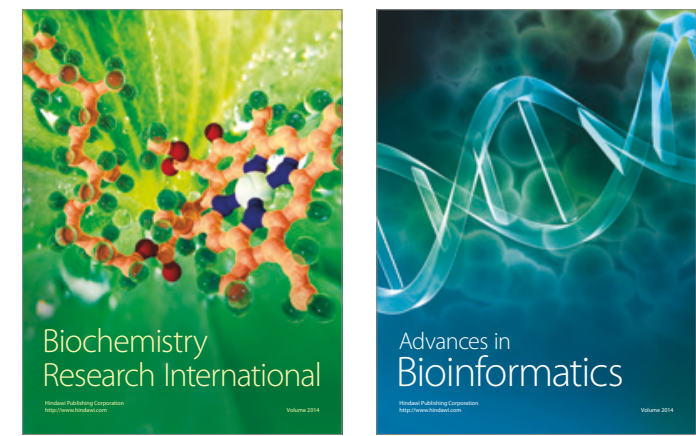

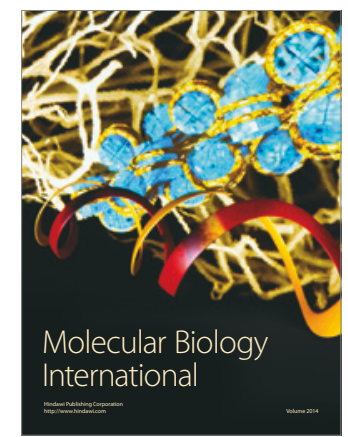

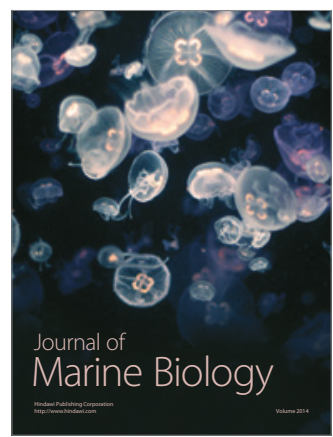

